\title{
TRANSITIVITY ON WEIERSTRASS POINTS
}

\author{
Zoë Laing and David Singerman \\ University of Southampton, School of Mathematics \\ Southampton SO17 1BJ, United Kingdom; zelaing@googlemail.com \\ University of Southampton, School of Mathematics \\ Southampton SO17 1BJ, United Kingdom; ds@maths.soton.ac.uk
}

\begin{abstract}
We search for Riemann surfaces whose automorphism groups act transitively on their Weierstrass points. One example is Klein's quartic. We find all hyperelliptic surfaces with this property, all surfaces with this property whose automorphism group is $P S L(2, q)$, many Platonic surfaces and other examples including Fermat curves. Basically, we find that the transitivity property seems quite rare and that the surfaces we have found with this property are interesting for other reasons too.
\end{abstract}

\section{Preliminaries}

Weierstrass Gap Theorem. [6] Let $X$ be a compact Riemann surface of genus $g$. Then for each point $p \in X$ there are precisely $g$ integers $1=\gamma_{1}<\gamma_{2}<\ldots<\gamma_{g}<$ $2 g$ such that there is no meromorphic function on $X$ whose only pole is one of order $\gamma_{j}$ at $p$ and which is analytic elsewhere.

The integers $\gamma_{1}, \ldots, \gamma_{g}$ are called the gaps at $p$. The complement of the gaps at $p$ in the natural numbers are called the non-gaps at $p$. Thus $\alpha$ is a non-gap at $p$ if there is a meromorphic function on $X$ that has a pole of order $\alpha$ at $p$ and is analytic on $X \backslash\{p\}$. If $f$ has a pole of order $\alpha$ at $p$, and $g$ has a pole of order $\beta$ at $p$, then $f g$ has a pole of order $\alpha+\beta$ at $p$ so that the non-gaps at $p$ form a semi-group under addition.

Definition 1. The weight of $p$, denoted by $w_{p}$, is given by

$$
w_{p}=\sum_{i=1}^{g}\left(\gamma_{i}-i\right) .
$$

Definition 2. $p$ is called a Weierstrass point if its weight is non-zero. Alternatively, $p$ is a Weierstrass point if there is a meromorphic function on $X$ with a pole of order $\leq g$ at $p$ and analytic elsewhere. (Thus $\alpha_{1} \leq g$ or $\gamma_{g}>g$ at $p$.)

Theorem 1. [6]

$$
\sum_{p \in X} w_{p}=g^{3}-g
$$

doi:10.5186/aasfm.2012.3711

2010 Mathematics Subject Classification: Primary 30F10, 14H55, $20 B 25$.

Key words: Compact Riemann surfaces, Weierstrass points, Fuchsian groups, finite automorphism groups. 
Theorem 2. [6] The number $|W|$ of Weierstrass points on $X$ (of genus $g \geq 2$ ) obeys

$$
2 g+2 \leq|W| \leq g^{3}-g
$$

Furthermore, $X$ has $2 g+2$ Weirstrass points if and only if $X$ is hyperelliptic (a two sheeted covering of the sphere) and the branch points are the Weierstrass points, (this follows from section 3). Generically, a Riemann surface of genus $g$ will have $g^{3}-g$ Weierstrass points (see Rauch [20]).

A useful method of showing that $p$ is a Weierstrass points is the following theorem whose proof can be found in [6] as Theorem V.1.7.

Theorem 3. (Schoeneberg) Let $h$ be an automorphism of a compact Riemann surface of genus $g \geq 2$. If $h$ fixes more than 4 points, then these fixed points are Weierstrass points.

This is equivalent to a theorem first stated by Schoeneberg in [22]. In this form it is due to Lewittes [14].

A compact Riemann surface $X$ of genus $g \geq 2$ can be represented as $\mathcal{U} / K$ where $K$ is a torsion free Fuchsian group (a surface group). The full group of automorphisms of $X$ is then $N(K) / K$, and it is well known that $N(K)$ is a Fuchsian group, and so a group $G$ of automorphisms of $X$ has the form $\Gamma / K$, where $\Gamma$ is a cocompact Fuchsian group. We call $\Gamma$ the lift of $G$.

Because of Schoeneberg's theorem we want to know the number of fixed points of an automorphism. For this we need a Theorem of Macbeath [17]. Rather than stating this theorem in full generality we will give a few consequences, also pointed out in [17]. The following result is useful.

Theorem 4. Let $G$ be a cyclic group of automorphisms of $X$ of order $n$ and represent $G$ as $\Gamma / K$ as above. Suppose that $\Gamma$ has periods $m_{1}, \ldots, m_{r}$. If $t \in G$ has order $d$, then the number $F(t)$ of fixed points of $t$ is

$$
F(t)=n \sum_{d \mid m_{i}} \frac{1}{m_{i}} .
$$

In section 4 we will use automorphism groups of the form $\operatorname{PSL}(2, q)$, where $q=p^{n}$, $p$ a prime.

Theorem 5. Let Aut $X \cong \operatorname{PSL}(2, q), q$ odd and again represent Aut $X=\Gamma / K$ as above. If $t \in$ Aut $X$ has order $d$ and $q$ is odd, then

$$
F(t)= \begin{cases}(q-1) \sum_{d \mid m_{i}} \frac{1}{m_{i}} & \text { if } d \mid \frac{q-1}{2} \\ (q+1) \sum_{d \mid m_{i}} \frac{1}{m_{i}} & \text { if } d \mid \frac{q+1}{2} \\ \frac{(n, 2)}{2} p^{n-1}(p-1) \sum_{m_{i}=p} 1 & \text { if } d=p .\end{cases}
$$


If $q$ is even, then

$$
F(t)= \begin{cases}2(q-1) \sum_{d \mid m_{i}} \frac{1}{m_{i}} & \text { if } d \mid q-1, \\ 2(q+1) \sum_{d \mid m_{i}} \frac{1}{m_{i}} & \text { if } d \mid q+1, \\ 2^{n-1} \sum_{m_{i}=2} 1 & \text { if } d=2 .\end{cases}
$$

\section{Maps on surfaces}

It is now useful to introduce maps on surfaces. We think of the word map in its geographic sense. Basically we just decompose the surface into simply-connected regions.

A formal theory was given in [9], although the origins of the theory go much further back. A map on a surface $X$ is an embedding of a graph $\mathcal{G}$ into $X$ such that the components of the complement of $\mathcal{M}$, which are called the faces of $\mathcal{M}$, are simply-connected. The detailed theory is set out in [9] but for briefer versions see [25] or [10]. There it is shown that if a surface carries a map, then it has the structure of a Riemann surface. A dart of a map $\mathcal{M}$ is a directed edge and the automorphism group of the map is also an automorphism group of the associated Riemann surface.

Definition 3. A map is regular if the automorphism group of the map acts transitively on the darts of the map.

The theory of regular maps goes back to the end of the 19th century; for a survey of the classical work see [5].

Definition 4. [25] A geometric point of a map is a vertex, face, centre or edgecentre.

Definition 5. A Riemann surface that contains a regular map is called Platonic. points.

In [25] we investigated the question of when the geometric points are Weierstrass

Platonic surfaces are those that are of the form $\mathcal{U} / K$ where $K$ is a torsion-free normal subgroup of a Fuchsian triangle group, with one period equal to 2, so that the automorphism group of a Platonic surface is an image of a $(2, m, n)$ triangle group. Two very famous Platonic surfaces are

(1) Klein's Riemann surface of genus 3. This surface has automorphism group $\operatorname{PSL}(2,7)$ of order 168 . It corresponds to a map of type $\{3,7\}$.

(2) Bring's surface of genus 4. This surface has automorphism group $S_{5}$ of order 120. It corresponds to a map of type $\{5,4\}$ which gives the small stellated dodecahedron. For Bring's surface, see [28].

These surfaces will appear again in section 7 .

Since Grothendieck's esquisse d'un programme, maps are known as clean dessins d'enfants. Grothendieck pointed out that Belyi's Theorem implies that the Riemann surface underlying a map corresponds to an algebraic curve defined over the field of algebraic numbers. 


\section{Hyperelliptic surfaces}

Hyperelliptic surfaces are Riemann surfaces that are two-sheeted coverings of the Riemann sphere. They admit an automorphism $J$, called the hyperelliptic involution, that interchanges the two sheets and which is central in the automorphism group of the surface. By the Riemann-Hurwitz formula there are $2 g+2$ branch points on the surface which are fixed by $J$. If the genus of the surface is greater than 1 then these branch points are the Weierstrass points on the surface (see $[6,111.7]$ ), or just use Schoeneberg's Theorem. We want to find the cases where the automorphism group of a hyperelliptic surface acts transitively on the $2 g+2$ Weierstrass points. The group then acts transitively on the projections of these Weierstrass points on the sphere. We study the cases where these points are vertices, face centres or edge centres of regular maps on the sphere. These are the Platonic solids. A study of branched coverings of the Platonic solids has been made by Jones and Surowski [11], and we use their results. (Except they considered branching over face-centres, whereas we consider branching over vertices.) First, some notation. If $\mathcal{M}$ is a platonic solid (or any map on a surface), we let $v, e$, and $f$ denote the number of vertices, edges and faces of $\mathcal{M}$. We let $m$ be the valency of a vertex, and $n$ the valency of a face, that is the number of edges of the face. The type of the solid is then $\{n, m\}$.

If we take a double cover, branched over the vertices we get a regular map of type $\{n, 2 m\}$, with $v$ vertices, $2 f$ faces and $2 e$ edges. The vertex valency is $2 m$ and the face valency is $n$ and so the map has has type $\{n, 2 m\}$. As the $v$ vertices are the branch points we can compute the genus $g$ from the formula $2 g+2=v$, that is

$$
g=(v-2) / 2
$$

or just by the Euler formula.

We note the following result which follows from Proposition 4 of [11]. Consider the Riemann surface as a double covering of a platonic solid with automorphism group $G$, which is branched over the vertices. Then the automorphism group of the surface (and of the lifted map) is $C_{2} \times G$ if the vertex valency is odd. We now consider the Platonic solids in turn.

(1) The cube, type $\{4,3\}, v=8, f=6, e=12$, so the double cover branched over the vertices has type $\{4,6\}$. It has 8 vertices, 12 faces and 24 edges. The vertices are the Weierstrass points so that $2 g+2=8$ giving $g=3$, which can also be found using the Euler characteristic. The genus of the surface is 3 and its automorphism group is $C_{2} \times S_{4}$.

The results for other double covers, branched over the vertices is similar and we just give the results, for details see [11].

(2) The tetrahedron, type $\{3,3\}$. The double cover branched over the vertices has type $\{3,6\}$; it has 4 vertices, 8 faces and has 12 edges and is of genus 1 . Its automorphism group is $C_{2} \times A_{4}$ of order 24 . It has genus 1 .

(3) The octahedron, type $\{3,4\}$. The double cover branched over vertices has type $\{3,8\}$; it has 6 vertices, 16 faces and 24 edges and genus 2 . Its automorphism group is $\mathrm{GL}(2,3)$ of order 48 (for the only group of automorphisms of a surface of genus 2 of order 48 is $\operatorname{GL}(2,3)$ ).

(4) The dodecahedron, type $\{5,3\}$. The double cover branched over the vertices has type $\{5,6\}$; it has 20 vertices, 24 faces and 60 edges It is of genus 9 . Its automorphism group is $C_{2} \times A_{5}$. 
(5) The icosahedron, type $\{3,5\}$. The double-cover branched over the vertices has type $\{3,10\}$; It has 12 vertices and 40 faces and 60 edges It is of genus 5 . Its automorphism group is $C_{2} \times A_{5}$.

(6) The dihedron $\Delta$, type $\{n, 2\}$. This solid has $n$ equally spaced vertices on the equator. It has $n$ edges and two faces, the upper and lower halves of the sphere. The double cover branched over the vertices has type $\{n, 4\}$. It has $n$ vertices, 4 faces and $2 n$ edges. Its genus is $(n-2) / 2$. The automorphism group of the Riemann surface that we obtain is twice as large as that of $D_{n}$ and so is equal to $4 n=8(g+1)$. These are the well known Accola-Maclachlan surfaces. (The order of the largest automorphism group $M(g)$ of a Riemann surface of genus $g$ obeys $M(g) \geq 8(g+1)$; the lower bound is always attained by the Accola-Maclachlan surfaces, $[1,18]$.) The automorphism group has presentation $\left\langle r, s \mid r^{4}=s^{n}=(r s)^{2}=\left(r^{-1} s\right)^{2}=1\right\rangle$.

(7) The hosohedron, type $\{2, n\}$. This is the dual of the dihedron. It has 2 vertices, $n$ edges and $n$ faces. The double cover branched over the vertices has type $\{2,2 n\}$ and has 2 vertices, $2 n$ edges and $2 n$ faces and genus 0 . Its automorphism group is $D_{2 n}$ if $n$ is even and $C_{2} \times D_{n}$ if $n$ is odd.

Branching over face-centres. By considering the dual we see that we get the same set of Riemann surfaces as we get by branching over faces.

Branching over edge-centres. We just find the genera of the double covers branched over the edge-centres. If there are $e$ edges in the Platonic solid then the double cover branched over these edge-centres also has $e$ edge-centres so that $e=2 g+2$. We thus find that the double covers branched over the edge-centres have genus $g=2$, for the tetrahedron, $g=5$ for the cube or octahedron $g=14$, for the icosahedron or dodecahedron.

(8) Star maps. A free edge of a map is an edge, which is not a loop, that only has one vertex. The star map $\mathcal{S}_{e}$ is a map on the sphere with one vertex $p, 1$ face and $e$ free edges which all have $p$ as its only vertex. If we draw the map so that the angles between its edges are all equal, then its automorphism group is $C_{e}$ which acts transitively by rotation on the edges. If we consider the double cover branched over the $e$ edge-centres, then its genus is $g=(e-2) / 2$. Its automorphism group is $C_{2} \times C_{e}$ if $e$ is odd and $D_{e}$ if $e$ is even. In both cases its order is $2 e=4 g+4$. Now this is half the order that we got when we considered branching over the vertices of of a dihedron. As these edge-centres are the vertices of a regular $n$-gon, we get the same set of Riemann surfaces as in (6), the Accola-Maclachlan surfaces.

A Fuchsian group interpretation. We briefly mention how this can be interpreted using Fuchsian groups. First of all we state the following result which is due to Maclachlan [18].

Theorem 6. $X=\mathcal{U} / K, K$ a surface group is hyperelliptic if and only if $K$ is a subgroup of index 2 in a Fuchsian group $\Gamma$ of signature $\left(0 ; 2^{2 g+2}\right)$, where the notation indicates $2 g+2$ periods equal to 2 . The quotient $\Gamma / K$ acts as the hyperelliptic involution.

We just consider one example, the octahedron with automorphism group $\operatorname{GL}(2,3)$ as in (3) above. It lifted to a map of type $\{3,8\}$ which lies on a Riemann surface $X=$ $\mathcal{U} / K, K$ a surface group. Let $j$ denote the hyperelliptic involution. By the results 
in [9] or [5] there is a homomorphism $\theta: \Gamma \rightarrow \operatorname{GL}(2,3)$ where $\Gamma$ has presentation $\left\langle x, y, z \mid x^{3}=y^{8}=(x y)^{2}=1\right\rangle$. If $j$ denotes the hyperelliptic involution (which is represented by $-I \in \mathrm{GL}(2,3))$, then by standard Fuchsian group results [23], it can be shown that $\theta^{-1}(\langle j\rangle)$ is a Fuchsian group of signature $\left(0 ; 2^{6}\right)$ which contains $K$ with index 2 so that $X$ is hyperelliptic by Maclachlan's Theorem.

Having found these hyperelliptic surfaces whose automorphism group acts transitively on the Weierstrass points we now show that these are all the surfaces with this property.

So suppose that $G<$ Aut $X$ acts transitively on the Weierstrass points of a hyperelliptic surface $X$ with hyperelliptic involution $J$. Then $X /\langle J\rangle=\Sigma$. The Weierstrass points on $X$ lower to $2 g+2$ points on $\Sigma$, which are permuted transitively by a finite subgroup of Aut $\Sigma$ which is well-known to be $\operatorname{PSL}(2, \mathbf{C})$. The finite subgroups of $\operatorname{PSL}(2, \mathbf{C})$ are the finite rotation groups of the sphere which are the ones that appear above in $(1), \ldots,(8)$. Thus there are no more hyperelliptic surfaces with transitive groups of automorphisms.

We sum up in

Theorem 7. For every integer $g \geq 1$ there is a hyperelliptic surface of genus $g$ whose automorphism group acts transitively on the Weierstrass points. These surfaces are the Accola-Maclachlan surfaces described in (6). If we assume that the branch points lie over the geometric points (see definition 4) of a Platonic solid, the only other hyperelliptic surfaces with transitive automorphism groups have genera $1,2,3,5,9$ and 14 which occur in the cases (1) to (5) above, and the last from the covering of the icosahedron branched over the edge-centres. As all these surfaces are covers of regular solids branched over the vertices or edge-centres they are unique. (Note: we get two surfaces of genus 5. The first is the two-sheeted cover of the icosahedron branched over the 12 vertices, the second being the two-sheeted cover of the cube branched over the edge-centres.)

We should also consider the case where he branch points do not lie over a geometric point of a platonic solid. In this case the finite rotation group of the solid acts freely on the projections of these branch points and so the number of branch points is $|G|$, where $G$ is the rotation group of the solid. For the tetrahedron, cube or icosahedron, this gives 12, 24, or 60 branch points, giving further hyperelliptic surfaces of genus 5,11 , or 29 with a transitive automorphism group. If for the solid we choose the dihedron $\Delta_{n}$ we get $g=n-1$ giving other surfaces of arbitrary genus, some of which will be Accola-Maclachlan.

\section{Surfaces with automorphism group $\operatorname{PSL}(2, q)$}

If $G$ is a group of automorphisms of a Riemann surface $X$ of genus $g \geq 2$, then the size of its automorphism group Aut $X$ is bounded above by $84(g-1)$. This is by a classic theorem of Hurwitz. The surfaces for which this bound is attained are called Hurwitz surfaces and their automorphism groups are known as Hurwitz groups. It is well known that $G$ is a Hurwitz group if and only if there is a homomorphism from the $(2,3,7)$ triangle group onto $G$. The smallest Hurwitz group is PSL $(2,7)$ of order 168; the corresponding surfaces is Klein's surface, which corresponds to the Klein quartic

$$
x^{3} y+y^{3} z+z^{3} x=0 .
$$


In [16] Macbeath proved

Theorem 8. Let $q$ be a prime power. The group $\operatorname{PSL}(2, q)$ is a Hurwitz group if and only if

(i) $q=7$ or

(ii) $q=p \equiv \pm 1(\bmod 7)$ or

(iii) $q=p^{3}$, where $p \equiv \pm 2$ or $\pm 3(\bmod 7)$.

Thus there are infinitely many surfaces that admit Hurwitz groups as their automorphism groups, and for this reason it is worth us studying the transitivity problem for Riemann surfaces whose automorphism group is $\operatorname{PSL}(2, q)$. However, there are many other Hurwitz groups. For example, Conder [2] showed that the alternating group $A_{n}$, is Hurwitz for all $n \geq 168$. The first 3 Hurwitz groups are of the form $\operatorname{PSL}(2, q)$, namely for $q=7,8,13$, corresponding to genera $3,7,14$. The next largest is of order 1344 which acts on a surface of genus 17 .

Theorem 9. If $q>15$, then the Hurwitz group PSL $(2, q)$ does not give a transitive action on the Weierstrass points of a Riemann surface that it defines.

By Theorem 5, the number of fixed points of an element of order 3 is greater than 5 , whereas the number of fixed points of an element of order 2 is greater than 7 and hence by Schoeneberg's Theorem these fixed points are Weierstrass points. Hurwitz groups are associated to a regular map on a surface, so the fixed points of elements of order 3 are vertices of the associated map, whereas the elements of order 2 are edge-centres. As $\Gamma(2,3,7)$ is maximal, the automorphism group of the surface and the automorphism group of the map coincide. As no automorphism of the map can take vertices to edge centres, the automorphism group of the surface does not act transitively on the Weierstrass points.

The cases $q=7,8$ and 13 are still to be dealt with.

Firstly, if $q=7$, then the corresponding Riemann surface of genus 3 is the wellknown Klein surface. Transitivity on the Weierstrass points was proved in [25] as the 24 Weirstrass points are shown to be the face-centres of the regular map of type $\{7,3\}$ on the surface. It is also proved in [19] by more group-theoretic methods. We just use a counting argument similar to [19]. This will also work for $q=8$ and is applicable to $q=13$ as also noted in [19].

Theorem 10. Let $W$ denote the set of Weierstrass points on the Klein surface $X$ of genus 3. The automorphism group $\operatorname{Aut}(X)$ acts transitively on $W$.

Proof. As $g=3$, the total weight of the Weierstrass points on $X$ is $3^{3}-3=24$ by Theorem 1 . Now $\operatorname{PSL}(2,7)$ besides acting as a group of automorphisms $X$ also acts as a group of automorphisms of the regular map of type $\{3,7\}$ underlying $X$. The only points with non-trivial stabilizers are the vertices, face-centres and edgecentres, where the stabilizers have orders 3,7 and 2 respectively. Thus the size of the possible orbits are $\sigma_{1}=168 / 7=24, \sigma_{2}=168 / 3=56, \sigma_{3}=168 / 2=84$ and $\sigma_{4}=168 / 1=168$, the latter case occurring for interior points of the faces of the map, i.e non-geometric points. If we let $w_{1}, w_{2}, w_{3}, w_{4}$ denote the weights of the Weierstrass points in these 4 orbits, we get

$$
\sum_{i=1}^{4} w_{j} \sigma_{j}=24,
$$


or

$$
24 \sigma_{1}+56 \sigma_{2}+84 \sigma_{3}+168 \sigma_{4}=24 \text {. }
$$

The only solution is $\sigma_{1}=1, \sigma_{2}=\sigma_{3}=\sigma_{4}=0$. Thus only the face-centres are Weierstrass points, and they all have weight 1 , and so Aut $X$ is transitive on the 24 Weierstrass points.

The next largest Hurwitz group is $\operatorname{PSL}(2,8)$ of order 504, and this acts on a surface of genus 7, often called Macbeath's surface. (Macbeath wrote an important paper on this Riemann surface, [15].)

Theorem 11. Let $W$ denote the set of Weierstrass points of Macbeath's surface $X$ of genus 7. The automorphism group of $X$ acts transitively on $W$.

Proof. The total weight of the Weierstrass points is $7^{3}-7=336$. We follow the proof of Theorem 10, we now get the equation

$$
\sum_{i=1}^{4} w_{j} \sigma_{j}=336
$$

or

$$
72 w_{1}+168 w_{2}+252 w_{3}=336 .
$$

The only solution is $w_{2}=2, w_{1}=w_{3}=0$, and so only vertices are Weierstrass points of weight 2 and Aut $X$ acts transitively on the Weierstrass points.

The next largest Hurwitz group is $\operatorname{PSL}(2,13)$ of order 1092. This acts as a group of automorphisms of a surface of genus 13. The total weight of the Weierstrass points is $14^{3}-14=2730$. Again, we follow the proof of Theorem 10 and we get

$$
156 w_{1}+364 w_{2}+546 w_{3}+1092 w_{4}=2730 .
$$

This has 10 solutions in positive integers; These are $\left(w_{1}, w_{2}, w_{3}, w_{4}\right)=$
(1) $(0,0,5,0)$
(2) $(0,0,1,2)$
(3) $(0,0,3,1)$
(4) $(0,3,3,0)$
(5) $(0,6,1,0)$
(6) $(7,0,3,0)$
(7) $(14,0,1,0)$
(8) $(0,3,1,1)$
(9) $(7,0,1,1)$
(10) $(7,3,1,0)$

If the first case occurs, then PSL $(2,13)$ acts transitively on the Weierstrass points which are all edge-centres of weight 5 , but if cases $2-7$ occur, there are two orbits of Weierstrass points, while if cases 8-10 occur, then there are 3 orbits. We cannot decide which of these occur so as in [19], we cannot decide transitivity. In fact it is possible that there are one, two, or three orbits of Weierstrass points. (However, because of the large number of solutions of the above equation we might guess that transitivity does not occur.) We can now easily deduce 
Theorem 12. If $X$ is a Hurwitz surface of genus 14 , on which PSL $(2,13)$ acts as a group of automorphisms (there are 3 of them [16]), then the edge-centres of the corresponding map of type $\{7,3\}$ are Weierstrass points.

Proof. By Theorem 5, the number of fixed points of an element of order 2 is 6 , so by Schoeneberg's Theorem these are Weierstrass points. Alternatively, in all the above solutions, $w_{3} \neq 0$.

So even though we cannot decide transitivity we know where 546 of the Weierstrass points are.

Added note. Very recently, Manfred Streit in a detailed investigation of the genus 14 Hurwitz surfaces has shown that the vertices and face-centres of he corresponding map are not Weierstrass points so that $w_{1}=w_{2}=0$. Thus only the first 3 of the above possibilities can occur, so the chances of transitivity have increased from one in ten to one in three! Also, there are at most two orbits of Weierstrass points.

By Theorems 9, 10 and 11 we see that only for the Macbeath-Hurwitz surfaces of genus 14 are we, as yet, unable to decide transitivity.

Macbeath [16] has shown that for all $q \neq 9$, that $\operatorname{PSL}(2, q)$ is an image of $\operatorname{PSL}(2, \mathbf{Z})$, the modular group which is a free product of $C_{2}$ and $C_{3}$. Thus $\operatorname{PSL}(2, q)$ is an image of the triangle group $(2,3, t)$ for some $t \geq 7$. Let $K_{t, q}$ be the kernel of the homomorphism $\theta:(2,3, t) \rightarrow \operatorname{PSL}(2, q)$ and let $X_{t, q}=\mathcal{U} / K_{t, q}$.

Theorem 13. If $q>15$ or $q=11$ or $q=t=13$, then Aut $X_{t, q} \cong \operatorname{PSL}(2, q)$ does not act transitively on $X_{t, q}$.

Proof. If $q>15$, then the proof follows as in theorem 9. If $q=11$, then by Theorem 5 we see that the number of fixed points of both an element of order 2 and of order 5 is equal to 5 , and so by Theorem 3, the edge-centres and face-centres of the corresponding map are Weierstrass points and so Aut $X$ does not act transitively. If $q=t=13$, then by Theorem 5 the number of fixed points of the element of order 13 is 6 and so again edge-centres and face-centres are Weierstrass points.

For $p$ prime let $\Gamma(p)$ be the principal congruence subgroup of the modular group $\Gamma$ of level $p$ and let the modular surface $X(p)$ be the compactification of $\mathcal{U} / \Gamma(p)$. Then $X(p)=X_{p, p}$, so by Theorems 10 and 13 we have

Corollary 14. Aut $X(p)$ acts transitvely on the Weierstrass points of $X(p)$ if and only if $p=7$.

\section{Platonic surfaces of low genus}

In [25] the following problem was investigated: When is a Weierstrass point on a Platonic surface a geometric point? We solved this for $g=2,3,4$ and, except for one possible exception, for $g=5$. This one exception corresponded to a map of type $\{15,6\}$ where we found that the vertices and face-centres were Weierstrass points, but we could not decide about the edge centres. (In [25], $\{m, n\}$, meant vertex valencies $m$ and face valencies $n$, unlike this paper.) We found the following results which we present as in the following two examples. 


\begin{tabular}{llllllcc}
\hline & & g & $V$ & $F$ & $E$ & $G$ & order of $G$ \\
\hline (i) & $\{12,3\}$ & 3 & $4^{2}$ & $16^{1}$ & 24 & $\langle\langle 2,3 \mid 3\rangle\rangle$ & 48 \\
(ii) & $\{8,3\}$ & 3 & $12^{2}$ & 32 & 48 & $(2,3,8 ; 3)$ & 96 \\
\hline
\end{tabular}

In (i) this means that the map type is $\{12,3\}$, the genus is 3 , there are 4 faces which are Weierstrass points of weight 2 , there are 16 vertices of weight 1 , and the edges are not Weierstrass points. As there are Weierstrass points at both vertices and face-centres (or, alternatively as they have different weights), we do not get transitivity on the Weierstrass points.

In (ii), we find that all of the Weierstrass points are at the face-centres, so that, as the automorphism group permutes the faces transitively, we get a transitive action on the Weierstrass points, The automorphism group $G$ is usually described in the Coxeter-Moser notation [5], but if the surface is an Accola-Maclachlan surface (see section 3 (6) where we give the presentation) we denote the group by $A M$; it has order $8(g+1)$.

We now examine the other cases in [25], where all the Weierstrass points are of the same type. We have to be careful of the fact that it is possible for a Riemann surface to carry more than one map (see [26]). This is when there is an inclusion relation between triangle groups, for example $(2, n, n)<(2,4, n)$, so that when a surface carries a regular map of type $(n, n)$ it may also carries one of type $(4, n)$. Also $(2, n, 2 n)<(2,3,2 n)$, (see [24]). We then choose the larger group.

We find the following cases:

\begin{tabular}{llllllcc}
\hline & & $\mathrm{g}$ & $V$ & $F$ & $E$ & $G$ & order of $G$ \\
\hline$(1)$ & $\{6,4\}$ & 2 & $6^{1}$ & 4 & 12 & $A M$ & 24 \\
$(2)$ & $\{8,3\}$ & 2 & 16 & $6^{1}$ & 24 & $\mathrm{GL}(2,3)$ & 48 \\
$(3)$ & $\{8,4\}$ & 3 & $8^{3}$ & 4 & 16 & $A M$ & 32 \\
$(4)$ & $\{6,4\}$ & 3 & 12 & $8^{3}$ & 24 & $S_{4} \times C_{2}$ & 48 \\
$(5)$ & $\{8,3\}$ & 3 & 32 & $12^{2}$ & 48 & $\left\langle x, y \mid x^{2}=y^{3}=(x y)^{8}=[x, y]^{3}=1\right\rangle$ & 96 \\
$(6)$ & $\{7,3\}$ & 3 & 56 & $24^{1}$ & 84 & $\mathrm{PSL}(2,7)$ & 168 \\
$(7)$ & $\{10,4\}$ & 4 & $10^{6}$ & 4 & 20 & $A M$ & 40 \\
$(8)$ & $\{5,4\}$ & 4 & 30 & 24 & $60^{1}$ & $S_{5}$ & 120 \\
$(9)$ & $\{12,4\}$ & 5 & $12^{10}$ & 4 & 24 & $A M$ & 48 \\
$(10)$ & $\{3,10\}$ & 5 & 40 & $12^{10}$ & 60 & $C_{2} \times A_{5}$ & 120 \\
$(11)$ & $\{8,3\}$ & 5 & 64 & $24^{5}$ & 96 & $\Gamma / \Gamma(8)$ & 192 \\
$(12)$ & $\{5,4\}$ & 5 & $40^{3}$ & 32 & 80 & $\left\langle x, y \mid x^{5}=y^{4}=(x y)^{2}=\left(x y^{-1}\right)^{4}=1\right\rangle$ & 160 \\
\hline
\end{tabular}

Now (1), (2), (3), (4), (7), (9) and (10) correspond to hyperelliptic surfaces and so have already appeared in section 3. Also (6) is Klein's surface which appeared in section 4 . The cases (5), (8), (11) and (12) give new examples where transitivity occurs. ((5) is the Fermat curve $F_{4}$, (see section 10), (8) is Bring's surface, (11) is a double cover of the Fermat curve, sometimes known as Wiman's curve and (12) is known as Humbert's curve. For these curves see [13]. For more details about the group in (12) see [4, p. 135]. This group is an elementary abelian group of order 16 semidirect product with $D_{5}$. For more details on the regular map and the group see $[4$, p. 135$]$. 


\section{Simple Weierstrass points}

A Weierstrass point of weight 1 is called a simple Weierstrass point. By equation 1, if all Weierstrass points of a surface of genus $g$ have weight 1 , then the number of Weierstrass points is equal to $g^{3}-g$. As shown in [20] in some sense this is the general case. However the following result shows that this is certainly not the case for Riemann surfaces whose automorphism group is transitive on the Weierstrass points.

Theorem 15. If there is a transitive action on the Weierstrass points on a Riemann surface $X$ of genus $g>2$ and the Weierstrass points are simple, then either $g=4$ and $X$ is Bring's surface, or $g=3$ and $X$ is Klein's surface or $g=3$ and Aut $X \cong S_{4}$. If the latter case occurs, then Aut $X$ acts freely on the Weierstrass points.

Proof. Let $|W|$ denote the number of Weierstrass points on $X$. As these Weierstrass points form an orbit under the action of $G=$ Aut $X$ then $|W|\left|G_{p}\right|=|G| \leq$ $84(g-1)$, where $G_{p}$ is the stabilizer of a Weierstrass point $p$ so that $|W| \leq 84(g-1)$. By Theorem 2, $|W|=g^{3}-g$. Hence $g^{3}-g \leq 84(g-1)$ and so $g \leq 8$. In general, transitivity implies that $|W| \leq M(g)$, the order of the largest group of automorphism for a surface of genus $g$. We now note that if $X=\mathcal{U} / K$ ( $K$ a surface group) has large enough automorphism group, then $X$ must be platonic. For Aut $X \cong \Gamma / K$ and if $\mid$ Aut $X \mid>24(g-1)$, then by the Riemann-Hurwitz formula, the measure of a fundamental region for $\Gamma$ is $<1 / 12$ and so $\Gamma$ must be a $(2, m, n)$ triangle group so that $X$ is platonic. So the largest automorphism groups are automorphism groups of regular maps, so we can consult [3] where we find that $M(6)=150, M(7)=504$, (for the Macbeath surface) and $M(8)=336$. However, $6^{3}-6=210>150$, and $8^{3}-8=504>336$ so we do not get transitivity for $g=6$ or 8 . If $g=7$, then the largest automorphism group is 504 which gives Macbeath's surface, the unique surface of genus 7 with 504 automorphisms, [16]. But we saw in the proof of Theorem 11 that the weights of the Weierstrass points of Macbeath's surface are 2 and so are not simple. The next highest order for an automorphism group in genus 7 is 288 corresponding to a $(2,3,8)$ group. As $288<7^{3}-7$ we cannot have transitivity.

Thus the genus of $X$ is $2,3,4$ or 5 . As $X$ is platonic we can use the lists in [25] to get information about the Weierstrass points.

(1) If $g=5$, then $|G|=\left(5^{3}-5\right)\left|G_{p}\right|=120\left|G_{p}\right|$. The surface $X$ must then be platonic. From [25] we find that $|G|=120$ and the map has type $\{3,10\}$ and the Weierstrass points have weight 10. (In fact this surface is the twosheeted covering of the icosahedron branched over the vertices that we found as example (5) in section 4.)

(2) If $g=4$, then $|G|=60\left|G_{p}\right|$. From [25] we find that either $|G|=120$ or $|G|=60,\left(G\right.$ being $S_{5}$ or $A_{5}$.) In the first case the map has type $\{4,5\}$ and is the map underlying Bring's surface. In the second case we get a map of type $\{5,5\}$ on the the same surface. This is because of the inclusion relationship $(2,5,5)<(2,4,5)$, and so the first map is a truncation of the second map. (See [26].) By [25] we see that the 60 edge centres are simple Weierstrass points.

(3) If $g=3$, then $|G|=24\left|G_{p}\right|$. If the surface is platonic, then again we consult [25] and we find that we must have $|G|=168$, the map has type $\{3,7\}$ so the surface is Klein's surface. However, we cannot guarantee that $X$ must be 
Platonic. A possibility is that $|G|=24, G \cong S_{4}$ and $G$ lifts up to a Fuchsian group of signature $\{2,2,2,3\}$. If this were to occur, then $G$ would permute the Weierstrass points freely. Also, as the complex dimension of the Teichmüller space of $\{0,2,2,2,3\}$ groups is equal to 1 there will be an uncountable number of surfaces of genus 3 admitting $S_{4}$ as a group of automorphisms. It is unclear whether there could be any where the group does not fix any Weierstrass points or whether the weight of the Weierstrass points is equal to one.

\section{A necessary condition for transitivity}

Let $G$ be a group of automorphisms of a Riemann surface $X$. Then $G$ lifts to a Fuchsian group $\Gamma$ with periods $m_{1}, \ldots, m_{r}$, corresponding to elliptic elements $x_{i}(i=$ $1, \ldots, r)$ with fixed points $a_{i}(i=1, \ldots, r)$. Furthermore, there is a homomorphism $\phi: \Gamma \rightarrow G$ with torsion-free kernel. All points of $\mathcal{U}$ with non-trivial stabilizer have the form $\gamma a_{i},(\gamma \in \Gamma)$ this point being stabilized by $\gamma x_{i} \gamma^{-1}$ of order $m_{1}$. The point $\gamma a_{i}$ projects to a point in $X$ stabilized by a cyclic group of order $m_{i}$, and all fixed points have this form. Now suppose that $G$ acts transitively on the the Weierstrass points $W$ of $X$. Then $W$ is an orbit so that

$$
|W|\left|G_{p}\right|=|G|
$$

where $G_{p}$ is the stabilizer of $p$, so that $\left|G_{p}\right|=m_{i}$, one of the periods of $\Gamma$.

As all points of $W$ have the same weight $w$, it follows from Theorem 1 , that

$$
|W| w=g^{3}-g
$$

We can therefore deduce

Theorem 16. If $G$ acts transitively on the Weierstrass points, then

$$
w=\frac{\left|G_{p}\right|\left(g^{3}-g\right)}{|G|} .
$$

(Thus the weight of a Weierstrass point is calculated in group theoretic terms! However we do have the group theoretic condition of transitivity.)

Example. If $G$ is a Hurwitz group of order $84(g-1)$, a homomorphic image of a triangle group $(2,3,7)$, then $m_{i}$ is one of 2,3 or 7 , so that if $G$ acts transitively on the Weierstrass points then $g(g+1)$ is divisible by 12,28 or 42 .

For example, the Hurwitz group of order 1344 which acts on a surface of genus 17 , does not act transitively. Unfortunately, this does not tell us anything about $\operatorname{PSL}(2,13)$ acting on a surface of genus 14 .

\section{Bi-elliptic surfaces}

A Riemann surface $X$ is called $\gamma$-elliptic if $X$ admits an involution $J$ such that $X /\langle J\rangle$ has genus $\gamma$. 0-hyperelliptic is just hyperelliptic, and 1-hyperelliptic is called bi-elliptic. To investigate transitivity on bi-elliptic surfaces we need the following theorems of Kato [12] and Garcia [7]. 
Theorem 17. (Kato) Let $X$ be a non-hyperelliptic Riemann surface of genus $g \geq 3$. Then for every point $p$ of $X$,

$$
0 \leq w_{p} \leq \begin{cases}\frac{g(g-1)}{3} & \text { if } g=3,4,6,7,9,10 \\ \frac{g^{2}-5 g+10}{2} & \text { otherwise }\end{cases}
$$

where $w_{p}$ is the weight of $p$.

Kato also exhibited a link between this bound and bi-elliptic surfaces.

Theorem 18. Let $X$ be a Riemann surface of genus $g \geq 11$. Then $X$ is bi-elliptic if and only if there exists $p \in X$ with weight $w_{p}$ such that

$$
\frac{g^{2}-5 g+6}{2} \leq w_{p}<\frac{g^{2}-g}{2} .
$$

In this case the possible values of $w_{p}$ are $\frac{g^{2}-5 g+6}{2}$ or $\frac{g^{2}-5 g+10}{2}$.

Garcia [7] proved that both these bounds are attained.

Theorem 19. Let $X$ be a bi-elliptic Riemann surface of genus $g \geq 11, g \neq 15$. If there exists $p \in X$ such that $w_{p}=\frac{g^{2}-5 g+10}{2}$ or $w_{p}=\frac{g^{2}-5 g+6}{2}$, then Aut $X$ does not act transitively on the Weierstrass points $W$ of $X$.

Proof. We prove this by contradiction so suppose that Aut $X$ does act transitively on $W$. If $w_{p}=\frac{g^{2}-5 g+10}{2}$ then all Weierstrass points of $X$ have this weight. Thus $\frac{g^{2}-5 g+10}{2}$ divides $g^{3}-g$ the quotient being $|W|$. Thus

$$
|W|=2 g+10+\frac{28 g-100}{g^{2}-5 g+10} .
$$

Therefore $\nu(g)=\frac{28 g-100}{g^{2}-5 g+10}$ must be an integer. However, $\nu(g)$ decreases for $g>11$ and for $g=11, \nu(g)<3$ and so $\nu(g)=1$ or 2 . There is no integral solution if $g=1$ and $\nu(g)=2$ implies that $g=4$ or $g=15$. Thus for $g>11, g \neq 15$ we cannot get a transitive action.

Similarly $\frac{g^{2}-5 g+6}{2}$ cannot divide $g^{3}-g$ for $g \geq 11$.

We thus have

Theorem 20. Let $X$ be an bi-ellipticsurface of genus $g>11, g \neq 15$. Then Aut $X$ does not act transitively on the Weierstrass points of $X$.

Using similar results of Garcia [7] we can also show that with the possible exception of a small number of values of $g$, that no 2-hyperelliptic surface of genus $g$ can have an automorphism group that acts transitively on the Weierstrass points.

\section{Fermat surfaces}

The Fermat surface $\mathbf{F}_{n}$ is the Riemann surface of the projective algebraic curve

$$
\left.\{x, y, z) \mid x^{n}+y^{n}+z^{n}=0\right\} .
$$

Let $\Gamma(n, n, n)$ be the triangle group with the presentation $\langle x, y, z| x^{n}=y^{n}=z^{n}=$ $x y z=1\rangle$. If we abelianize this group, we get $\mathbf{Z}_{n} \oplus \mathbf{Z}_{n}$ so that the commutator subgroup $K_{n}$ has index $n^{2}$. It is known that $\mathbf{F}_{\mathbf{n}} \cong \mathcal{U} / \mathbf{K}_{\mathbf{n}},[10]$. We then find that $K_{n}$ has genus $(n-1)(n-2) / 2$. As $K_{n}$ is characteristic in $\Gamma(n, n, n)$ and as $\Gamma(n, n, n)$ 
is normal in the maximal triangle group $\Gamma(2,3,2 n)$ with $\Gamma(2,3,2 n) / \Gamma(n, n, n) \cong S_{3}$, it follows that Aut $F_{n} \cong\left(\mathbf{Z}_{n} \oplus \mathbf{Z}_{n}\right) \rtimes S_{3}$

We now describe how $\left(\mathbf{Z}_{n} \oplus \mathbf{Z}_{n}\right) \rtimes S_{3}$ acts on the points of the Fermat curve $\mathbf{F}_{n}$. We first define an action of $\mathbf{Z}_{n}^{3}$ on $\mathbf{F}_{n}$. If $(a, b, c) \in \mathbf{Z}_{n}^{3}$ and $(x, y, z) \in \mathbf{F}_{n}$, we let $(a, b, c) \wedge(x, y, z)=\left(e^{2 \pi i(a / n)}, e^{2 \pi i(b / n)}, e^{2 \pi i(c / n)}\right)$. The kernel of this action consists of the set of points $(a, b, c)$ where $a=b=c$ which form a group isomorphic to $\mathbf{Z}_{n}$ and so if we factor out this subgroup we get an action of $\mathbf{Z}_{n} \oplus \mathbf{Z}_{n}$ as a group of automorphisms of $\mathbf{F}_{n}$. Also there is an action of $S_{3}$ by permuting the coordinates and so we find an action of $\mathbf{F}_{n} \cong\left(\mathbf{Z}_{n} \oplus \mathbf{Z}_{n}\right) \rtimes S_{3}$ as a group of automorphisms of $\mathbf{F}_{n}$.

Weierstrass points on the Fermat curve. Let $\alpha=e^{2 \pi i / n}$ and $\beta=e^{\pi i / n}$. Then the $3 n$ points of $\mathbf{F}_{n}$ of the form

$$
\left(0, \alpha^{j}, \beta\right),\left(\beta, 0, \alpha^{j}\right),\left(\alpha^{j}, \beta, 0\right)
$$

$(j=0, \ldots, n-1)$, are called the trivial points of $\mathbf{F}_{n}$ and Hasse [8] showed that these points are Weierstrass points of weight

$$
(n-1)(n-2)(n-3)(n+4) / 24 .
$$

When $n=4$ this is equal to 2 and so the total weight is 24 . By (2) in section 1 , this shows that these are all the Weierstrass points of $\mathbf{F}_{4}$. For $n \geq 5$ there are more Weierstrass points, called the Leopoldt points [21]. These are the $3 n^{2}$ points of the form $\left(\gamma, \beta_{1}, \beta_{2}\right)$, where $\gamma^{n}=2, \beta_{1}^{n}=\beta_{2}^{n}=-1$. According to Towse [27], their weight is $\geq(n-1)(n-3) / 8$ if $n$ is odd and $(n-2)(n-4) / 8$ if $n$ is even and this inequality is an equality if $n \leq 8$.

When $n=5$, the total weight of the Leopoldt points is equal to 1 and so the total weights of the trivial points and the Leopoldt points is equal to $15 \cdot 9+75 \cdot 1=$ $210=6^{3}-6$ and so by Theorem 2 , the trivial points and the Leopoldt points include all of the Weierstrass points. When $n \geq 6$, a similar count shows that there must be further Weierstrass points.

Theorem 21. The automorphism group of $\mathbf{F}_{n}$ acts transitively on the Weierstrass points if and only if $n=4$.

Proof. We consider the action of the automorphism group of the Fermat curve defined above. Clearly the set of trivial points is an orbit. The set of Leopoldt points is also an orbit and these orbits are distinct. For $n=4$ the only Weierstrass points are the trivial ones and thus we get transitivity. For $n>4$, there are Weierstrass points of Leopoldt type and so we do not get transitivity on all the Weierstrass points.

Notes.

(1) As Rohrlich remarked [21], the automorphisms like

$$
(x, y, z) \mapsto(\alpha x, y, z)
$$

(or multiply the 2 nd or 3rd coordinates by $\alpha$ ) are automorphisms that fix $n$ trivial points and hence for $n>4$ we know these are Weierstrass points by Schoeneberg's Theorem. Also, we can see that the automorphim $(x, y, z) \mapsto$ $(x, z, y)$ fixes the Leopoldt points of the form $(\gamma, \beta, \beta)$ showing these are Weierstrass points. As all Leopoldt points form an orbit under Aut $\mathbf{F}_{n}$ we see that they are all Weierstrass points. 
(2) Because of the inclusion relationship $\Gamma(4,4,4)<\Gamma(2,3,8)$ we can deduce that $\mathbf{F}_{4}$ is the Platonic surface of type $\{8,3\}$ in section 5 .

\section{Conclusion}

We showed in Theorem 7 that there is one family of hyperelliptic surfaces, namely the Accola-Maclachlan surfaces, where the automorphism group acts transitively on the Weierstrass points. However for the families of non-hyperelliptic surfaces we have considered there seem to be few examples and the surfaces we have found which have this property such as the Klein surface, Bring's surface, the Macbeath surface, the Fermat curve $\mathbf{F}_{4}$ are important for other reasons. It would be interesting to search for other non-hyperelliptic Riemann surfaces whose automorphism group acts transitively on the Weierstrass points.

\section{References}

[1] Accola, R. D. M.: On the number of automorphisms of a closed Riemann surface. - Trans. Amer. Math. Soc. 131, 1968, 398-408.

[2] Conder, M. D. E.: The symmetric genus of alternating and symmetric groups. - J. Combin. Theory Ser. B 39:2, 1985, 179-186.

[3] Conder, M., and P. Dobcsányi: Determination of all regular maps of small genus. - J. Combin. Theory Ser. B 81:2, 2001, 224-242.

[4] Coxeter, H. S. M.: The abstract groups $G^{m, n, p}$. - Trans. Amer. Math. Soc. 45:1, 1939, 73150.

[5] Coxeter, H. S. M., and W. O J. Moser: Generators and relations for discrete groups. Ergeb. Math. Grenzgeb. 14, 4th edition, Springer-Verlag, Berlin, 1980.

[6] Farkas, H. M., and I. Kra: Riemann surfaces. - Grad. Texts in Math. 71, Springer-Verlag, New York, 1980.

[7] García, A.: Weights of Weierstrass points in double coverings of curves of genus one or two. - Manuscripta Math. 55:3-4, 1986, 419-432.

[8] Hasse, H.: Mathematische Abhandlungen. Band 2. - Walter de Gruyter, Berlin-New York, 1975 (in German).

[9] Jones, G. A., and D. Singerman: Theory of maps on orientable surfaces. - Proc. London Math. Soc. (3) 37:2, 1978, 273-307.

[10] Jones, G. A., and D. Singerman: Bely functions, hypermaps and Galois groups. - Bull. London Math. Soc. 28:6, 1996, 561-590.

[11] Jones, G. A., and D. B. Surowski: Regular cyclic coverings of the Platonic maps. - European J. Combin. 21:3, 2000, 333-345.

[12] Kato, T.: Non-hyperelliptic Weierstrass points of maximal weight. - Math. Ann. 239:2, 1979, $141-147$.

[13] Keem, C., and G. Martens: On curves with all Weierstrass points of maximal weight. Arch. Math. 94, 2010, 339-349.

[14] Lewittes, J.: Automorphisms of compact Riemann surfaces. - Amer. J. Math. 85, 1963, $734-752$.

[15] Macbeath, A. M.: On a curve of genus 7. - Proc. London Math. Soc. (3) 15, 1965, 527-542.

[16] Macbeath, A. M.: Generators of the linear fractional groups. - In: Number Theory, Proc. Sympos. Pure Math., Vol. XII (Houston, Tex., 1969), Amer. Math. Soc., Providence, R.I., $1969,14-32$. 
[17] Macbeath, A. M.: Action of automorphisms of a compact Riemann surface on the first homology group. - Bull. London Math. Soc. 5, 1973, 103-108.

[18] Maclachlan, C.: A bound for the number of automorphisms of a compact Riemann surface. - J. London Math. Soc. 44, 1969, 265-272.

[19] Magaard, K., and H. Völklein: On Weierstrass points of Hurwitz curves. - J. Algebra $300: 2,2006,647-654$.

[20] RAUCH, H. E.: Weierstrass points, branch points, and moduli of Riemann surfaces. - Comm. Pure Appl. Math. 12, 1959, 543-560.

[21] Rohrlich, D. E.: Some remarks on Weierstrass points. - In: Number theory related to Fermat's last theorem (Cambridge, Mass., 1981), Progr. Math. 26, Birkhäuser, Boston, Mass., $1982,71-78$.

[22] Schoeneberg, B.: Über die Weierstrass-Punkte in den Körpern der elliptischen Modulfunktionen. - Abh. Math. Sem. Univ. Hamburg 17, 1951, 104-111 (in German).

[23] Singerman, D.: Subgroups of Fuschian groups and finite permutation groups. - Bull. London Math. Soc. 2, 1970, 319-323.

[24] Singerman, D.: Finitely maximal Fuchsian groups. - J. London Math. Soc. (2) 6, 1972, 29-38.

[25] Singerman, D., and P. D. Watson: Weierstrass points on regular maps. - Geom. Dedicata $66: 1,1997,69-88$.

[26] Singerman, D., and R. I. Syddall: The Riemann surface of a uniform dessin. - Beiträge Algebra Geom. 44:2, 2003, 413-430.

[27] Towse, C.: Weierstrass weights of fixed points of an involution. - Math. Proc. Cambridge Philos. Soc. 122:3, 1997, 385-392.

[28] Weber, M.: Kepler's small stellated dodecahedron as a Riemann surface. - Pacific J. Math. $220: 1,2005,167-182$.

Received 8 July 2011 